\title{
Das May-Thurner-Syndrom - der Beckenvenensporn - in heutiger Zeit
}

\section{May-Thurner syndrome - the pelvic vein spur - in modern times}

Autoren

Dieter Urbas', 2 , Erich Brenner ${ }^{2}$

Institute

1 Institut für Diagnostische und Interventionelle Radiologie, Landeskrankenhaus Feldkirch, Österreich

2 Institut für Klinisch-Funktionelle Anatomie, Medizinische Universität Innsbruck, Österreich

Schlüsselwörter

Beckenvenensporn, V. iliaca communis sinistra, Cockett-Syndrom, lliakalkompressionssyndrom

Key words

pelvic vein spur, left common iliac vein, Cockett's syndrome, iliac compression syndrome

online publiziert 29.04 .2021

Bibliografie

Phlebologie 2021; 50: 184-195

DOI 10.1055/a-1394-3111

ISSN 0939-978X

(C) 2021. Thieme. All rights reserved.

Georg Thieme Verlag KG, Rüdigerstraße 14,

70469 Stuttgart, Germany

Korrespondenzadresse

Ao. Univ. Prof. Dr. Erich Brenner, MME (Bern)

Institut für Klinisch-Funktionelle Anatomie

Medizinische Universität Innsbruck, Müllerstrasse 59,

6020 Innsbruck, Österreich

Erich.Brenner@i-med.ac.at

\section{ZUSAMMENFASSUNG}

Hintergrund Venensporne in der linken V. iliaca communis sind eine bekannte Entität, insbesondere im Zusammenhang mit schweren tiefen Beckenvenenthrombosen. Allerdings sind fundierte Kenntnisse über diese Sporne nur spärlich vorhanden. Es wurden 5 Formen von Venenspornen beschrieben: zentrale Sporne, Adhäsionen, Brücken, Klappen und Bänder. Dennoch sind sowohl die Lokalisation als auch die Position von venösen Spornen nicht angemessen untersucht worden. Material/Methoden 101 Körper (68 Frauen, 33 Männer), die dem Institut für Klinisch-Funktionelle Anatomie mit informierter Zustimmung vermacht wurden, wurden untersucht. Ein Individuum musste aufgrund einer Tumorinfiltration ausgeschlossen werden.

Der Startpunkt (mp0) wurde am kaudalsten Teil des Zusammenflusses der beiden gemeinsamen Beckenvenen definiert,

der distalste Punkt (mp5) an der Mündung der linken aufsteigenden Lumbalvene. Nach der Teilung der Länge in 4 Viertel wurden Dicke und Umfang an jedem Messpunkt gemessen. Die Klassifizierung der Sporne wurde nach Pinsolle et al. (1982) durchgeführt.

Ergebnisse Die Wanddicke nahm vom Startpunkt (Mittelwert: $0,21 \mathrm{~mm}$ ) zum ersten Viertel (Mittelwert: 0,16 mm) ab und dann bis zum vierten Viertel (Mittelwert: 0,24 mm) kontinuierlich wieder zu. Der Umfang war am Startpunkt maximal (Mittelwert: 45,45 mm), nahm zum zweiten Viertel hin ab (Mittelwert: $38,14 \mathrm{~mm}$ ) und nahm dann bis zum vierten Viertel wieder zu (Mittelwert: 39,32 mm).

Insgesamt fanden wir am Zusammenfluss 125 Sporne; im ersten, rechten Viertel 23 Sporne, im zweiten Viertel 18 Sporne, im dritten Viertel 11 Sporne, und im linken, vierten Viertel auch noch 4 Sporne. Zwei Individuen wies keinen venösen Sporn auf.

Schlussfolgerung Zentrale venöse Sporne treten unmittelbar am venösen Zusammenfluss auf. Sie könnten Überreste von ostialen Klappen sein, während die anderen Typen andere Ursachen haben könnten. Zum Beispiel kann eine Verwachsung aus der embryologischen Entwicklung stammen, was durch den Fund unterschiedlicher Wandstärken unterstützt wird. Auch ohne Kompression der linken V. iliaca communis fanden wir erhebliche Umfangsunterschiede, die zu deutlichen Unterschieden in den Kalibern führten. Während der Embryogenese bildet sich der proximale Teil der linken V. iliaca communis als anastomotisches interkardinales Netzwerk kleiner Venen, die im Laufe der weiteren Entwicklung verschmelzen. Die Annahme eines unterschiedlichen embryonalen Ursprungs der verschiedenen Viertel, entweder Kardinalvene oder venöses anastomotisches Netzwerk, wird durch die Unterschiede sowohl in der Dicke der Venenwand als auch im Venenumfang unterstützt. Mit Ausnahme der kaudalen zentralen Sporne am Zusammenfluss stellen sie grundsätzlich ein Strömungshindernis dar und können so einen prädisponierenden Faktor für eine tiefe Venenthrombose darstellen. Diese Strukturen finden sich zudem nicht nur am Zusammenfluss, sondern auch über die ganze Länge der interkardinalen Anastomose. Demnach erscheint ein ursächlicher Zusammenhang mit einer Kompression durch die A. iliaca communis dextra unwahrscheinlich. 


\section{ABSTRACT}

Background Venous spurs in the left common iliac vein are a well-known entity, especially in the context of severe deep pelvic vein thrombosis. However, sound knowledge of these spurs is sparse. Five forms of venous spurs have been described: central spurs, adhesions, bridges, valves, and bands. Nevertheless, both the localization and position of venous spurs have not been adequately studied.

Material/methods 101 bodies (68 females, 33 males) bequeathed to the Institute for Clinical and Functional Anatomy with informed consent were studied. One individual had to be excluded due to tumour infiltration.

The starting point $(\mathrm{mp} 0)$ was defined at the most caudal part of the two common iliac veins' confluence, the most distal point (mp5) at the mouth of the left ascending lumbar vein. After dividing the length into four quarters, thickness and circumference were measured at each measurement point. Classification of spurs was done according to Pinsolle et al. (1982). Results Wall thickness decreased from the starting point (mean: $0.21 \mathrm{~mm}$ ) to the first quarter (mean: $0.16 \mathrm{~mm}$ ) and then continuously increased again until the fourth quarter (mean: $0.24 \mathrm{~mm}$ ). The girth was maximum at the starting point (mean: $45.45 \mathrm{~mm}$ ), decreased towards the second quarter (mean: $38.14 \mathrm{~mm}$ ), and then increased again until the fourth quarter (mean: $39.32 \mathrm{~mm}$ ).
In total, we found 125 spurs at the confluence; in the first, right quarter 23 spurs, in the second quarter 18 spurs, in the third quarter eleven spurs, and the left, fourth quarter also four spurs. Two individuals had no venous spur.

Conclusion Central venous spurs occur solely at the venous confluence. They could be remnants of ostial valves, while the other types could have other causes. For example, an adhesion may originate from embryological development, supported by the finding of different wall thicknesses. Even without compression of the left iliac vein, we found considerable differences in circumference, resulting in marked differences in calibre. During embryogenesis, the left iliac vein's proximal part forms as an anastomotic intercardinal network of small veins that fuse during further development. The assumption of a different embryonic origin of the different quarters, either cardinal vein or venous anastomotic network, is supported by the differences in both vein wall thickness and venous circumference. Apart from the caudal central spurs at the confluence, they are fundamentally an obstruction to flow and maybe a predisposing factor for deep venous thrombosis. Moreover, these structures are found at the confluence and along the entire length of the intercardinal anastomosis. Accordingly, a causal relationship with compression by the right common iliac artery seems unlikely.

\section{Einleitung}

\section{Literaturübersicht}

Die Vereinigung der rechten und linken Venae iliacae communes und das Endsegment der linken V. iliaca communis (VICS) sind offensichtlich ein äußerst kritischer Bereich. Diese Einmündung ist eine Prädilektionsstelle für ausgedehnte Thrombembolien der Beckenvenen, die oft zu einer kompletten Obstruktion des Beckenvenensystems führen. Diese Obstruktion kann sich sogar bis in das proximale Bein ausdehnen oder zu einer Lungenembolie (Pulmonalarterienthrombembolie) führen. Mehrere neuere Studien zeigen, dass das Stenting der VICS deren Striktur korrigiert und die peripheren Refluxparameter verbessert [1].

Eine der meist diskutierten - und akzeptierten - Kausalketten beginnt mit einem venösen Sporn innerhalb der VICS. Während diese venösen Sporne seit Virchows Zeiten [2] immer wieder beschrieben wurden, war es der herausragende Verdienst von Robert May und Josef Thurner, ihre klinische Relevanz richtig zu erkennen und zu verbreiten [3], während andere Untersuchungen [4, 5] nie die Aufmerksamkeit erhielten, die sie verdient hätten.

McMurrich beschrieb 1908 angeborene Verwachsungen, welche die vordere und hintere Wand der VICS verschmolzen, was zu einer Verkleinerung oder Teilung ihres Lumens führte [4]. Er fand bei 107 Personen: (1) Säulen, die sich zwischen den beiden Wänden der Vene erstrecken (14,02\%), (2) entweder 3-eckige $(10,28 \%)$ oder eher lineare $(4,67 \%)$ Adhäsionen am lateralen
Rand der Vene und (3) 3-eckige Adhäsionen am medialen Rand der Vene $(1,87 \%)$.

Schilling berichtete 1926 über eigenartige Brückenbildungen im Bereich der V. iliaca communis und zum Teil auch in der V. iliaca externa, „die teils als geringe, quer zur Längsachse verlaufende Leisten der Venenwand ein geripptes Aussehen verliehen, teils die Lichtung quer durchzogen “ [6].

Ehrich und Krumbhaar stellten 1943 eine umfangreiche Studie über eine „häufige obstruktive Anomalie der Mündung der linken Vena iliaca communis“ vor [5]. Sie fanden eine Klappe der VICS an der Einmündung in die rechte V. iliaca communis (VICD) (einen zentralen Sporn) in 76,25\%. Interessant war, dass das Auftreten dieses zentralen Sporns mit zunehmendem Alter abnahm, oder wie die Autoren schrieben: „[...] it is 'worn off' in many persons with advancing age, and presumably worn down in still more." Sie klassifizierten 5 Gruppen von behindernden Läsionen innerhalb der VICS. Gruppe I zeigte alle Übergänge von einer schmalen Verdickung bis zu 3-eckig geformten Bereichen, die den oberen Pol des Zusammenflusses obstruieren (9,74\%). Gruppe II zeigte im Wesentlichen das gleiche Bild, außer dass alle einen Kanal aufwiesen, der entlang der linken Venenwand verlief; und einige hatten auch einen oder mehrere zusätzliche Kanäle, die durch den Hauptteil der obstruierenden Läsion verliefen (5,90\%). Gruppe III umfasste mehrere Arten von Adhäsionen, die das Lumen in 2 mehr oder weniger gleiche Teile teilten (3,08\%). Gruppe IV umfasste Adhäsionen am unteren Pol des Zusammenflusses, die die Tendenz zeigten, sich hinter die Klappe zu erstrecken (2,31\%). Gruppe V umfasste verschiedene Läsionen, von denen die meisten 
durch mehrere unregelmäßige Bänder gekennzeichnet waren (3,33\%). Die Breite des Lumens der VICS unmittelbar unter den Obstruktionen wurde durch deren Vorhandensein nicht wesentlich verändert, außer in 2 Fällen. Entsprechend ihrer Abbildungen befanden sich alle diese Obstruktionen an oder unmittelbar vor dem Zusammenfluss der VICS und der VICD. Die histologische Analyse zeigte eine große Menge an elastischem mit etwas kollagenem Gewebe und glatten Muskelzellen. Diese Autoren schlussfolgerten, dass die große Mehrheit der Obstruktionen nach der Geburt, hauptsächlich während der Entwicklung und des Wachstums, entsteht, aber nur wenige, wenn überhaupt, nach dem zweiten Lebensjahrzehnt gebildet werden. Hinsichtlich der Ursache der Obstruktionen kommen sie zu dem Schluss, dass der Druck der kreuzenden rechten A. iliaca communis die ordnungsgemäße Entwicklung der Mündung der VICS stört oder eine Verletzung verursachen könnte, die zu einer Organisation und Fibrose führt. Einige der Obstruktionen, die in Gruppe V enthalten waren, erwiesen sich als organisierte Thromben.

Robert May und Josef Thurner veröffentlichten 1956 ihre bahnbrechende Untersuchung über „ein[en] Gefäßsporn in der Vena iliaca communis sinistra als wahrscheinliche Ursache der überwiegend linksseitigen Beckenvenenthrombose“ [7]; ein Jahr später folgte dann eine entsprechende englischsprachige Veröffentlichung [8]. Hintergrund ihrer Untersuchungen war die auch damals schon bekannte Tatsache, dass Beckenvenenthrombosen mehrheitlich auf der linken Seite auftreten. Sie beschreiben auch gleich die „alte VIRCHowsche [...] Erklärung [2]: Normalerweise kreuzt die A. il. comm. dex. nach ihrem Abgang aus der Aorta abdominalis die V. il. comm. sin. an ihrer Vorderseite. Die auf die Vene drückende arterielle Pulswelle würde nun - so wird angenommen - den Blutfluß aus dem linken Bein hemmen, was als Argument zur Erklärung der ganzen Frage diente.“ [7]. Da diese Erklärung aber die beiden Autoren unbefriedigt ließ, untersuchten sie die Beckenvenen an 430 Verstorbenen. Dabei fanden May und Thurner (1) eine „in ihrer Auswirkung vom Grad der Ausbildung abhängige Abweichung [...] kulissenartige, zarte, von der medialen oder lateralen Wand wie ein Segel gegen die Lichtung vorragende und sie einengende Membran“ (medialer bzw. lateraler Venensporn; Sporn-Gruppe I); (2) eine „brückenpfeilerähnliche, in den Blutstrom ragende und ihn teilende Wehr [...], die meist in der Einzahl vorhanden, in der Mitte der Lichtung aufragt und ebenfalls streng dorso-anteriore Lage einnimmt" (zentraler Venensporn; Sporn-Gruppe II) und (3) ein „beinahe vollkommener Verschluss der V. il. comm. sin. an der Einmündung in die Vena cava inferior [...]. Die Vorder- und Hinterwand des Gefäßrohres ist meist bis auf einige mm einander genähert, das Restlumen von zahlreichen zarten Brücken mit dazwischen liegenden Spalten unterteilt, geradezu gezähnelt, so daß ein eigentümlicher wie abgesteppter Lichtungsspalt sich bietet“" (partielle Obliteration; Sporn-Gruppe III; - Tab. 1) [7]. May und Thurner schrieben auch, dass sie zwischen diesen Grundformen auch Zwischenstufen beobachten konnten. Niemals aber konnten sie derartige Veränderungen bei Föten oder Neugeborenen nachweisen ( $\triangleright$ Tab. 1).

Sie fanden aber eine Zunahme im höheren Lebensalter und eine häufigere Spornbildung bei Frauen. Interessant sind auch die histologischen Befunde: die „Sporne [waren] im Wesentlichen aus Fibrozyten mit reichlich Zwischensubstanz aufgebaut. Im Gewebe zahlreiche Kapillaren, die von Plasmazellen und Lymphozyten locker
- Tab. 1 Häufigkeiten der Beckenvenensporne nach May und Thurner (1956) [7].

\begin{tabular}{|l|l|r|r|}
\hline & & \multicolumn{1}{|c|}{$\mathbf{n}$} & $\%$ \\
\hline Erwachsene & & 430 & \\
\hline & Sporn-Gruppe I & 59 & $11 \%$ \\
\hline & Sporn-Gruppe II & 17 & $4 \%$ \\
\hline & Sporn-Gruppe III & 4 & $1 \%$ \\
\hline Embryonen und & & 88 & \\
\hline Neugeborene & & & \\
\hline & Sporn-Gruppen I-III & 0 & $0 \%$ \\
\hline
\end{tabular}

gesäumt sind. Eine Resorzinfuchsinfärbung zeigt, daß die elastischen Fasern der Venenwand nicht in die Spornbildung miteinbezogen sind, sondern eine begrenzende Schicht zwischen Vene und Sporngewebe darstellen. [...] Hämosiderin, das auf eine in Organisation sich befindende Thrombose schließen lassen könnte, wird nicht gefunden. " [7].

Die Gruppe um Frank B. Cockett untersuchte 1968 das schon 1965 von Cockett und Thomas [9] beschriebene „iliac compression syndrome“, auch Cockett-Syndrom genannt, genauer [10]. Im Phlebogramm fanden sie eine teilweise durchscheinende Einbuchtung am proximalen Ende der VICS, was als Kompression der Vene durch die darüber liegende A. iliaca communis dextra interpretiert wurde. Bänder oder Adhäsionen, welche die vordere und hintere Wand der VICS verbinden, fanden sich bei 14 von 100 Sektionen. Mit einer Länge von 1-5 mm ließen diese Veränderungen sich in 3 Gruppen einteilen: (1) nur ein seitlicher Lappen (6 Fälle), (2) ein zentrales Einzel- oder Doppelband (6 Fälle) und (3) ein fast vollständiger Verschluss des Venenlumens (2 Fälle). Die seitlichen Klappen und die Verschlussmembran waren in der Regel perforiert und hatten das Aussehen eines Fallgitters. Ihre histologischen Befunde unterscheiden sich jedoch grundlegend von denen von May und Thurner. Auch sie kommen zu dem Schluss, dass die Venensporne nicht aus der Organisation von Thromben bzw. Thrombemboli entstehen können; allerdings bleibt für sie die Genese zwischen den Thesen einer Akquisition und einer angeborenen Störung unentschieden.

Auch Chermet konnte 1979 die beiden wesentlichen Thesen nicht entscheiden, stellt sie aber ausführlich gegenüber [11].

Pinsolle und Mitarbeiter stellten 1982 eine weitere Typisierung mit 5 verschiedenen Formen von Venenspornen vor: zentraler Sporn (sp), Adhäsion (ad), Brücke (br), Klappe (kl) und Band (ba) [12].

Eine genauere histologische Untersuchung veröffentlichten Mitsuoka und Mitarbeiter 2014 [13]. In allen Spornen wurden $\alpha$-SMA-Immunfärbung-positive Strukturen festgestellt. Darüber hinaus waren in allen Fällen an beiden Enden der Sporne die Tunica media und die Membrana elastica interna vorhanden, die mit der Venenwand kontinuierlich waren. Andererseits wurden zahlreiche Variationen in den inneren Strukturen der Sporne festgestellt: Blutgefäße waren in einigen Spornen vorhanden, in anderen jedoch nicht. Elastische plattenartige Strukturen waren in Spornen mit einer Membrana elastica interna vorhanden, aber in ande- 
ren nicht. Die Tunica media der gegenüberliegenden Venenwand setzte sich nicht mit der inneren elastischen Lamina fort, aber setzte sich in Spornen ohne elastische plattenartige Struktur fort. In Spornen, in denen die Tunica media der Venenwand fortgesetzt wurde, setzte sich die Adventitia auch in einigen Spornen fort, in anderen jedoch nicht. Diese Befunde deuten darauf hin, dass es möglicherweise angeborene und erworbene Bildungsmechanismen gibt.

\section{Embryologische Grundlagen}

Die Venen der unteren Körperhälfte entwickeln sich aus einer zunächst bilateral symmetrischen Anlage, gefolgt von mehreren höchst komplizierten Interphasen [14]. Die primordialen kaudalen Kardinalvenen liegen dorsal des Mesonephros. Sie sammeln zunächst ausschließlich das Blut aus dem unteren Teil des Körpers. Wenn der Embryo etwa 10 mm CRL (crown-rump-length) erreicht, werden diese kaudalen Kardinalvenen nach kaudal bzw. distal durch die Sakrokardinalvenen ergänzt, die sich bis ins Becken und in die Beine fortsetzen. Diese Sakrokardinalvenen liegen im Gegensatz zu den zuvor erwähnten kaudalen Kardinalvenen dorsal der Nabelarterien. Mehrere Plexus-artige Anastomosen verbinden die beiden Sakrokardinalvenen. Diese Anastomosen nehmen die kaudalen Venen auf, die sich im weiteren Verlauf zur medianen Sakralvene oder -venen entwickeln. Die VICS wird zum größten Teil aus der Anastomose der beiden hinteren Kardinalvenen abgeleitet. Im Laufe der Entwicklung verschwindet die linke hintere Kardinalvene stromabwärts von dieser Anastomose allmählich. Die VICS wird also in ihrem ersten, distalen Teil von der linken hinteren Kardinalvene und in ihrem rechten, proximalen Teil von der hinteren interkardialen Anastomose gebildet, während die VICD vollständig aus dem distalen Teil der rechten hinteren Kardinalvene resultiert [11].

\section{Die Studie}

Während diese Übersicht zu zeigen scheint, dass viele Daten vorhanden sind, sind aber weder die genauere Position noch die Lokalisation der Venensporne innerhalb der VICS ausreichend untersucht worden. Wir haben uns daher entschieden, selbst diesbezügliche Untersuchungen anzustellen und dabei die Typisierung der Venensporne nach Pinsolle et al. [12] zu verwenden, da sie uns als die genaueste erscheint. Zudem haben wir die Untersuchung auf die gesamte Strecke der interkardialen Anastomose ausgedehnt.

\section{Material und Methode}

101 kaukasische Körperspender*innen (68 Frauen, 33 Männer) wurden untersucht. Ein männliches Exemplar wies eine starke Infiltration der VICS durch Krebs auf und wurde daher von dieser Studie ausgeschlossen. 96 Präparate wurden aus studentischen Sezierkursen gewonnen, die sorgfältig mit einer FormaldehydPhenol-Lösung konserviert wurden [15]; 4 Präparate wurden aus einem anderen Präparierkurs gewonnen und zunächst mit einer Alkohol-Glycerin-Lösung konserviert.

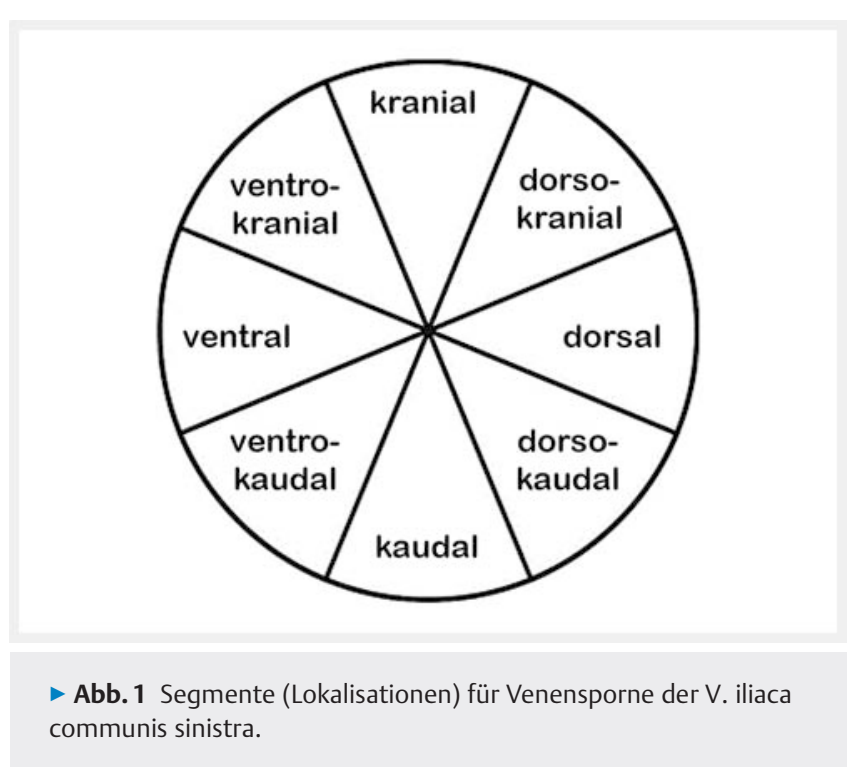

Die Körper wurden von Personen gespendet, die vor ihrem Tod ihre informierte Zustimmung zur Verwendung für wissenschaftliche und pädagogische Zwecke gegeben hatten [16]. Was die Repräsentativität betrifft, so hat eine kürzlich durchgeführte Analyse gezeigt, dass die gespendeten Körper eine repräsentative Stichprobe der allgemeinen österreichischen Bevölkerung im Sterbealter darstellen [17].

Aufgrund der unterschiedlichen Präparationen in den Sezierkursen war es zunächst notwendig, Strukturen in situ zu trennen und eine Übersicht mit anschließender Fotodokumentation zu erstellen. Diese Übersicht zeigte retroperitoneale Strukturen wie die Nieren, den Plexus lumbosacralis, beide Mm. iliopsoas, die Aorta mit ihren Ästen und die V. cava inferior $(\mathrm{VCI})$ mit den beiden Venae iliacae communes einschließlich ihrer venösen Äste. Nacheinander wurden die venösen Strukturen und ihre Äste vorsichtig mobilisiert und für die Entfernung vorbereitet. Die VCl wurde im Bereich der Nierenvenen abgetrennt, während die beiden Vv. iliacae communes distal der Vereinigung in V. iliaca externa bzw. V. iliaca interna exzidiert wurden. Jede gewonnene Probe wurde für die anschließende Fotodokumentation mit einer Formaldehyd-Phenol-Lösung gespült. Danach wurden alle Proben in einer Formaldehyd-Phenol-Lösung aufbewahrt, die zur Konservierung verwendet wurde. Zwischen Probenahme und Analyse wurde die Formaldehyd-Phenol-Lösung 2-mal ausgetauscht. Die Messungen wurden mit einem elektronischen Messschieber durchgeführt. Der Startpunkt (mp0) wurde an der kaudalsten Stelle der Vereinigung der VICD und VICS zur VCI (Zusammenfluss) definiert, der distalste Punkt (mp4) an der Mündung bzw. am Abgang der linken $\checkmark$. lumbalis ascendens. Durch diese Unterteilung ergaben sich 5 Messpunkte (mp0-mp4) und 4 Viertel zur genaueren Bestimmung der Lage der Venensporne. An jedem Messpunkt wurden die Dicke und der Umfang der VICS gemessen. Die Klassifizierung der Sporne nach Pinsolle wurde durch die Messung ihrer Höhe und Breite ergänzt [12]. Ihre Lokalisation wurde mit kranial, ventrokranial, dorsokranial, ventral, dorsal, ventrokaudal, dorsokaudal bzw. kaudal dokumentiert ( $\triangleright$ Abb. 1). 
- Tab. 2 Statistische Analyse der kontinuierlichen Variablen nach Geschlecht.

\begin{tabular}{|c|c|c|c|c|c|c|c|c|}
\hline & Position & Geschlecht & $\mathbf{n}$ & Missing & Mittelwert & SD & $95 \%-K I$ & t-test \\
\hline \multirow[t]{2}{*}{ Alter (Jahre) } & & q & 68 & - & 80,40 & 13,72 & $77,08-83,72$ & \multirow{2}{*}{$\begin{array}{l}t(98)=1,13 \\
p=0,261\end{array}$} \\
\hline & & $0^{x}$ & 32 & - & 77,29 & 10,65 & $77,29-81,13$ & \\
\hline \multirow[t]{2}{*}{ Größe (cm) } & & q & 60 & 8 & 160,18 & 7,03 & $158,37-162,00$ & \multirow{2}{*}{$\begin{array}{l}t(88)=-9,61 \\
p=0,000\end{array}$} \\
\hline & & $0^{x}$ & 30 & 2 & 175,00 & 6,61 & $172,53-177,47$ & \\
\hline \multirow[t]{2}{*}{ Länge der VICS (mm) } & & ㅇ & 68 & - & 54,50 & 8,80 & $52,37-56,63$ & \multirow{2}{*}{$\begin{array}{l}t(98)=1,29 \\
p=0,202\end{array}$} \\
\hline & & $0^{x}$ & 32 & - & 51,89 & 10,76 & $48,01-55,77$ & \\
\hline \multirow{10}{*}{$\begin{array}{l}\text { Dicke der Wand der } \\
\text { VICS }(\mathrm{mm})\end{array}$} & \multirow[t]{2}{*}{ mp0 } & ㅇ & 68 & - & 0,19 & 0,12 & $0,16-0,21$ & \multirow{2}{*}{$\begin{array}{l}t(98)=-2,54 \\
p=0,013\end{array}$} \\
\hline & & $0^{x}$ & 32 & - & 0,26 & 0,16 & $0,20-0,32$ & \\
\hline & \multirow[t]{2}{*}{ mp1 } & q & 68 & - & 0,15 & 0,06 & $0,14-0,17$ & \multirow{2}{*}{$\begin{array}{l}t(98)=-1,46 \\
p=0,146\end{array}$} \\
\hline & & $0^{x}$ & 32 & - & 0,17 & 0,07 & $0,15-0,20$ & \\
\hline & \multirow[t]{2}{*}{$\mathrm{mp2}$} & ㅇ & 68 & - & 0,16 & 0,08 & $0,14-0,18$ & \multirow{2}{*}{$\begin{array}{l}t(98)=-0,82 \\
p=0,413\end{array}$} \\
\hline & & $\sigma^{x}$ & 32 & - & 0,17 & 0,07 & $0,15-0,20$ & \\
\hline & \multirow[t]{2}{*}{$\mathrm{mp3}$} & ㅇ & 68 & - & 0,19 & 0,09 & $0,17-0,21$ & \multirow{2}{*}{$\begin{array}{l}t(98)=-0,17 \\
p=0,866\end{array}$} \\
\hline & & $0^{x}$ & 32 & - & 0,19 & 0,08 & $0,16-0,22$ & \\
\hline & \multirow[t]{2}{*}{ mp4 } & q & 68 & - & 0,24 & 0,12 & $0,21-0,27$ & \multirow{2}{*}{$\begin{array}{l}t(87,91)=-0,53, \\
p=0,598\end{array}$} \\
\hline & & $0^{x}$ & 32 & - & 0,25 & 0,08 & $0,22-0,28$ & \\
\hline \multirow[t]{10}{*}{ Umfang der VICS (mm) } & \multirow[t]{2}{*}{$\mathrm{mp0}$} & ㅇ & 68 & - & 45,78 & 10,68 & $43,20-48,37$ & \multirow{2}{*}{$\begin{array}{l}t(98)=0,49 \\
p=0,627\end{array}$} \\
\hline & & $0^{x}$ & 32 & - & 44,75 & 7,70 & $41,98-47,53$ & \\
\hline & \multirow[t]{2}{*}{$\mathrm{mp} 1$} & ㅇ & 68 & - & 37,52 & 7,80 & $35,63-39,41$ & \multirow{2}{*}{$\begin{array}{l}t(98)=-1,54 \\
p=0,127\end{array}$} \\
\hline & & $\sigma^{x}$ & 32 & - & 40,09 & 7,69 & $37,31-2,86$ & \\
\hline & \multirow[t]{2}{*}{$\mathrm{mp2}$} & q & 68 & - & 36,73 & 7,46 & $34,93-28,54$ & \multirow{2}{*}{$\begin{array}{l}t(98)=-2,69 \\
p=0,008\end{array}$} \\
\hline & & $0^{x}$ & 32 & - & 41,12 & 7,90 & $38,27-43,97$ & \\
\hline & \multirow[t]{2}{*}{ mp3 } & q & 68 & - & 37,40 & 7,56 & $35,57-39,23$ & \multirow{2}{*}{$\begin{array}{l}t(98)=-2,46 \\
p=0,015\end{array}$} \\
\hline & & $0^{x}$ & 32 & - & 41,29 & 6,93 & $38,79-43,79$ & \\
\hline & \multirow[t]{2}{*}{ mp4 } & + & 68 & - & 38,01 & 7,45 & $36,21-39,82$ & \multirow{2}{*}{$\begin{array}{l}t(98)=-2,44 \\
p=0,017\end{array}$} \\
\hline & & $\sigma^{\pi}$ & 32 & - & 42,11 & 8,59 & $39,07-45,20$ & \\
\hline
\end{tabular}

$\mathrm{VICS}=\mathrm{V}$. iliaca communis sinistra, $\mathrm{mp} 0=$ Zusammenmündung; $\mathrm{mp} 1$ = erstes Viertel; $\mathrm{mp}=$ zweites Viertel; $\mathrm{mp} 3$ = drittes Viertel; $\mathrm{mp} 4=$ viertes Viertel.

Die Abmessungen der Venensporne wurden zusätzlich mit einer elektronischen Schieblehre gemessen. Die Messung jedes einzelnen Parameters wurde zusätzlich fotodokumentiert.

Die Daten wurden je Präparat in einem eigenen Untersuchungsbogen schriftlich erfasst, zur Verarbeitung in Microsoft Excel 2010 (Microsoft Corporation, Redmond, WA, USA) übertragen und mit GNU PSPP Statistical Analysis Software, Release 1.2.0-g0fb4db (Free Software Foundation, Boston, MA, USA; [18]) ausgewertet. Als Signifikanzniveau haben wir einen Wert von 0,05 festgelegt.

\section{Ergebnisse}

Eine detaillierte statistische Analyse der kontinuierlichen Variablen findet sich in $>$ Tab. 2. Die untersuchten Probanden waren im Durchschnitt 79 Jahre alt (Mittelwert: 79,41 Jahre; SD: 12,844; 95 \%-KI: 76,86-81,96; kein Geschlechtsunterschied). Sie hatten eine durchschnittliche Körpergröße von 165,12 cm (SD: 9,81; 95 \%-KI: 163,07-167,18); Männer waren signifikant größer als Frauen. Die VICS war im Mittel 53,66 mm lang (SD: 9,49; $95 \%-K I$ : 51,78-55,55; kein Geschlechtsunterschied).

Die Wanddicke nahm von mp0 (Mittelwert: 0,21mm; SD: 0,14; $95 \%-K I$ : 0,18-0,24; signifikanter Geschlechtsunterschied) unmittelbar zum ersten Viertel (mp1; Mittelwert: 0,16mm; SD: 0,06; $95 \%$ KI: 0,15-0,17; kein Geschlechtsunterschied) ab und dann bis zum vierten Viertel wieder kontinuierlich zu (mp2: Mittelwert: 0,17mm; SD: 0,06; $95 \%-K I:$ 0,15-0-18; mp3: Mittelwert: 0,19mm; SD: 0,08; $95 \%-K I:$ 0,17-0,21; mp4: Mittelwert: 0,24mm; SD 0,10; $95 \%-\mathrm{KI}$ : 0,22-0,26; jeweils kein Geschlechtsunterschied) ( $\triangleright$ Abb. 2).

Der Umfang war am Zusammenfluss am größten (Mittelwert: 45,45mm; SD: 9,08; $95 \%-K I$ : 43,51-47,40; kein Geschlechtsunterschied), nahm zum zweiten Viertel hin ab (mp1: Mittelwert: 38,34mm; SD: 7,82; $95 \%-K I: 36,79-39,89$, kein Geschlechtsunterschied; mp2: Mittelwert: 38,14mm; SD: 7,84; $95 \%-K I: ~ 36,58-$ 


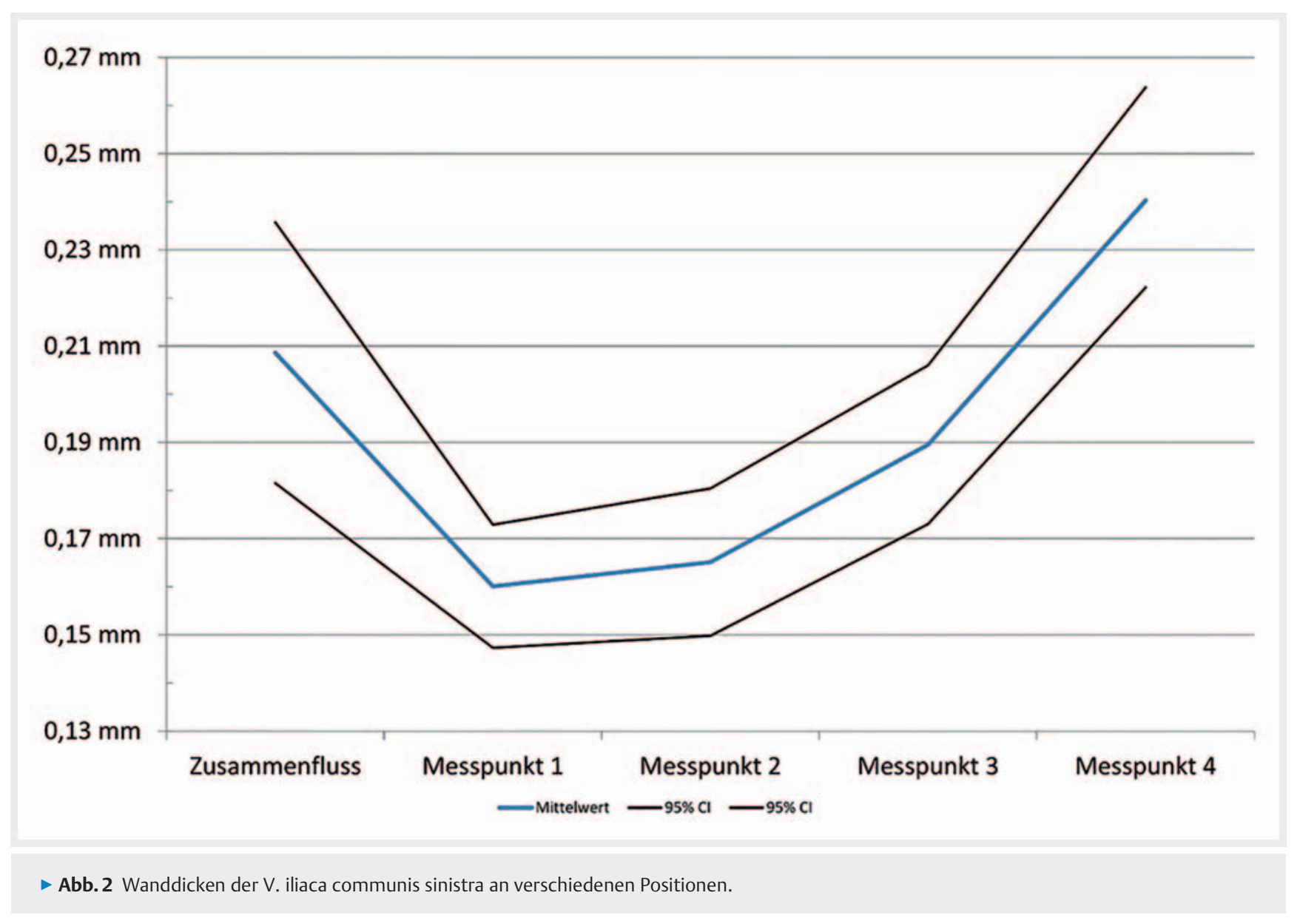

39,69; bei Männern signifikant größer), und stieg danach wieder an (mp3: Mittelwert: 38,64mm; SD: 7,55; $95 \%-K I: ~ 37,14-40,14$; mp4: Mittelwert: 39,32mm; SD: 8,02; $95 \%-K I: ~ 37,73-40,91$; bei Männern jeweils signifikant größer) ( $\triangleright$ Abb. 3).

Insgesamt identifizierten wir 185 Venensporne in insgesamt 98 von 100 untersuchten Individuen ( Tab. 3, 4, $\triangleright$ Abb. 4, 5), nur 2 Fälle (eine Frau, 97 á; ein Mann, 95 á) wiesen keinen Venensporn auf; in 2 weiteren Fällen ersteckte sich je ein Venensporn über mehr als ein Viertel.

Im Einzelnen beobachteten wir 125 Sporne am Zusammenfluss (79sp, 34ad, 1br, 2kl, 9ba); im ersten Viertel 23 Sporne (6ad, 10kl, 7ba), im zweiten Viertel 18 Sporne (6ad, 2br, 5kl, 5ba), im dritten Viertel 11 Sporne (1br, 6kl, 4ba), während das vierte Viertel 4 Sporne (2ad, $1 \mathrm{kl}, 1 \mathrm{ba}$ ) aufwies; zuzüglich fanden sich je 2 Adhäsionen, die sich vom ersten zum zweiten Viertel erstreckten, und 2 Brücken, die sich vom ersten bis zum vierten Viertel erstreckten $(\triangleright$ Tab. 5).

16 Männer und 32 Frauen wiesen nur einen Venensporn unterschiedlichen Typs auf, während die übrigen mehrere Kombinationen verschiedener Formen von Venenspornen aufwiesen (vergleiche Abb. 4).

\section{Diskussion}

Mit Sicherheit erreicht unsere Untersuchung nicht die Zahlen von Ehrich und Krumbhaar oder May und Thurner [5, 7], liegen aber in der Größenordnung der anderen Studien [4, 10, 12]; sie liefert aber ganz andere Ergebnisse. Mit Ausnahme von Pinsolle und Mitarbeitern ergaben die morphologischen Untersuchungen eine Inzidenz von gut $22 \%$ an Venenspornen [10]; Pinsolle und Mitarbeiter fanden in $68 \%$ Venensporne der einen oder anderen Form. In unseren 100 Individuen fanden wir in 98 zumindest einen Venensporn (98\%); einzig eine 97-jährige Frau und ein 95-jähriger Mann wiesen keinen Venensporn auf. Die meisten davon fanden sich im Bereich der Vereinigung der VICD mit der VICS (mp0), so wie in den früheren Studien beschrieben. Allerdings fanden wir auch links der Zusammenmündung bis hin zur Mündung bzw. zum Abgang der V. lumbalis ascendens sinistra noch weitere Venensporne; diese Bereiche der ursprünglich als hintere interkardianale Anastomose angelegten Teile der VICS wurden in den früheren Studien aber gar nicht untersucht. Bedauerlicherweise fehlen ausreichende Altersdaten aus den anderen, früheren Studien, um einen verlässlichen Zusammenhang zwischen dem Alter und der Anzahl von Venenspornen herstellen zu können. Dies wäre aber notwendig, um die Schlussfolgerungen von May und Thurner [7] oder der Gruppe um F. Cockett [10] weiter präzisieren zu können.

Diese hintere interkardinale Anastomose, also die VCIS, zeigt einige weitere interessante Besonderheiten. Sie besitzt einen Um- 


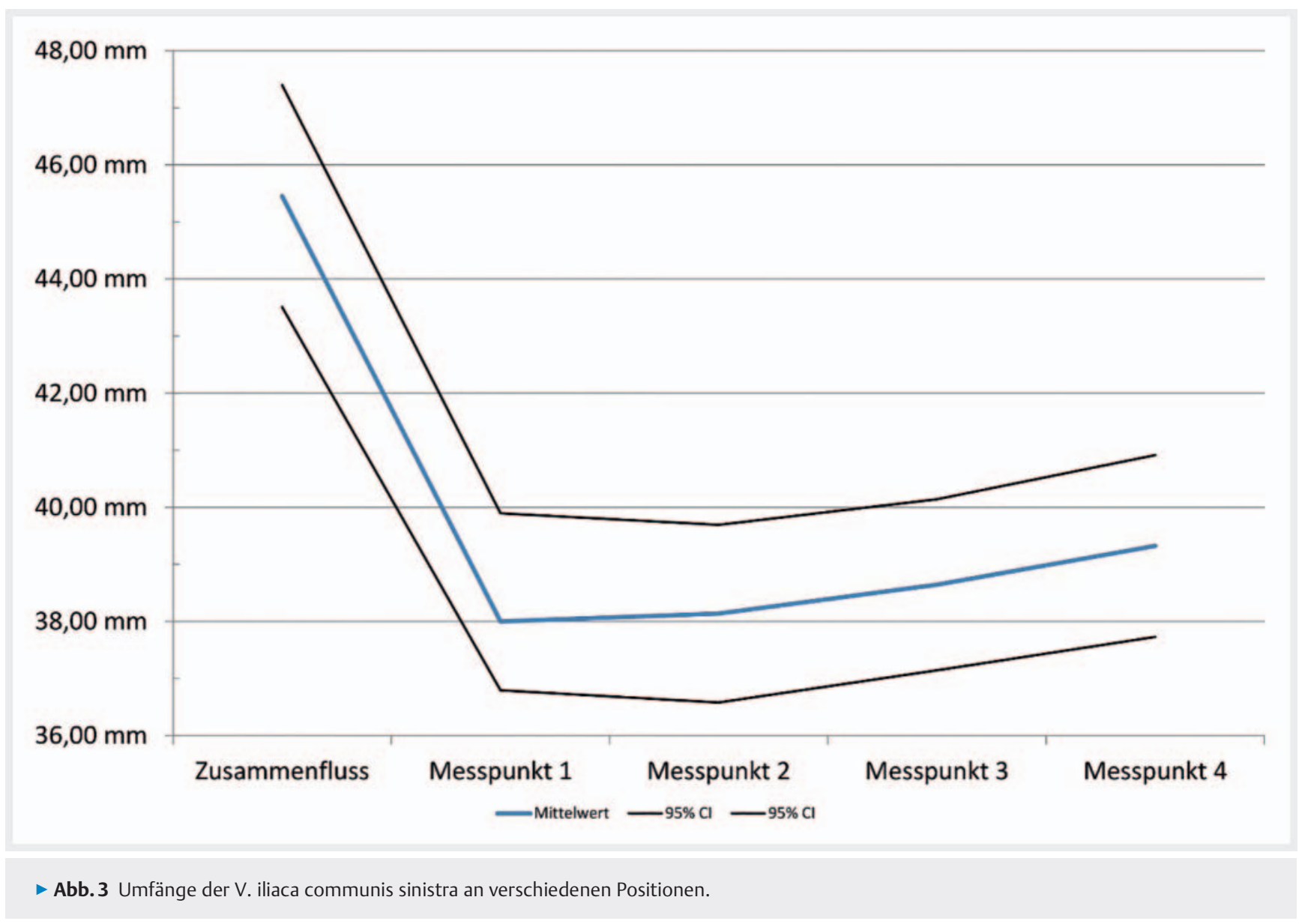

fang von etwa 39 mm, nur im Bereich der Vereinigung mit der VICD weitet sich das Gefäß auf durchschnittlich $45 \mathrm{~mm}$ signifikant auf. Anders sieht es mit den Wanddicken aus: das am Weitesten links liegende Viertel zeigt eine Wanddicke von 0,24 mm, diese geht dann auf $0,19 \mathrm{~mm}, 0,17 \mathrm{~mm}$ und $0,16 \mathrm{~mm}$ zurück, um sich im Bereich des Zusammenflusses dann auf 0,21 mm zu verdicken. Dies könnte seine Ursache eben in den unterschiedlichen embryologischen Ursprüngen der VICS-Abschnitte haben. Während der Embryogenese bildet sich der proximale oder terminale Teil der VICS als anastomotisches Netzwerk aus kleinen Venen, die beide Sakrokardinalvenen verbinden und im weiteren zeitlichen Verlauf normalerweise konfluieren. Während zentrale Sporne nur unmittelbar am Zusammenfluss der beiden gemeinsamen Beckenvenen zu finden sind, wurden die anderen Typen, Adhäsionen, Klappen, Brücken und Bänder, auch in den restlichen Vierteln nachgewiesen. Daher könnten die letztgenannten Typen Überbleibsel einer unvollständigen Konsolidierung dieses embryonalen anastomotischen Netzwerks sein. Die Annahme eines unterschiedlichen embryonalen Ursprungs der verschiedenen Viertel, entweder (Sakro-) Kardinalvene oder venöses anastomotisches Netzwerk, wird durch die Unterschiede sowohl in der Dicke der Venenwand als auch im Venenumfang unterstützt. Da diese Strukturen auch mehrfach vorkommen, ist ein kausaler Zusammenhang mit einer Kompression durch die querende A. iliaca communis dextra eher unwahrscheinlich, da sich diese Querung ganz überwiegend im rechten, terminalen Viertel der VCIS befindet. Die Querung durch

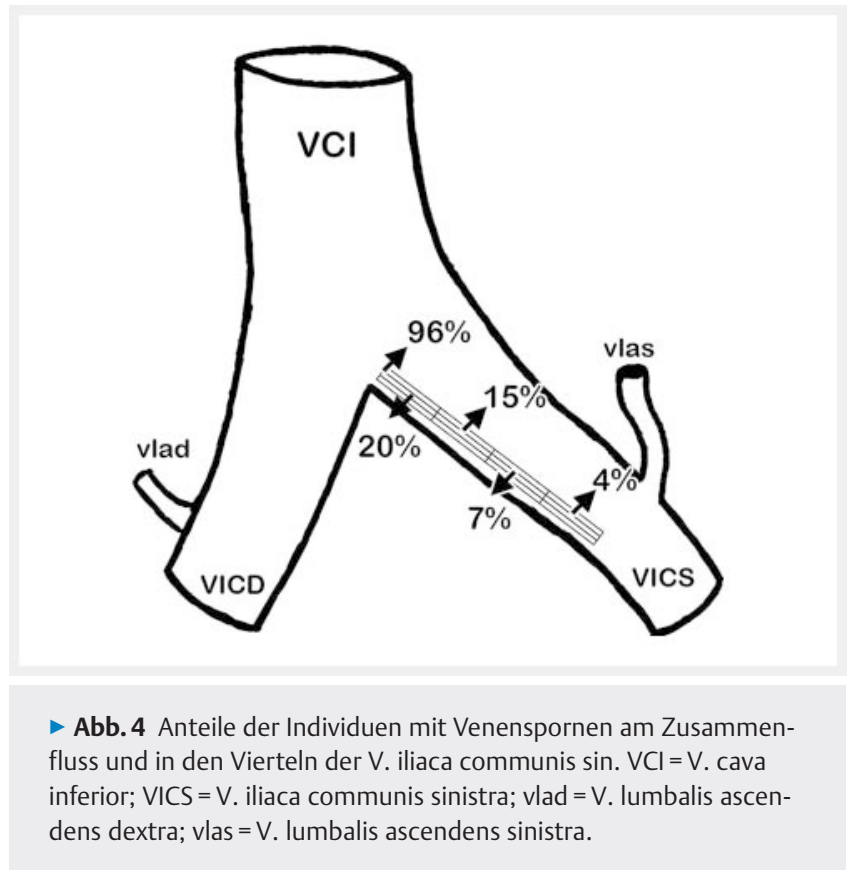

die A. iliaca communis sinistra [19] findet sich andererseits im ganz linken Viertel. Beide können somit nicht die Ursache für Beckenvenensporne in den beiden mittleren Vierteln darstellen. Wenn aber die Formation einer Adhäsion, einer Brücke oder eines 


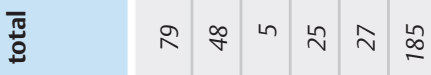

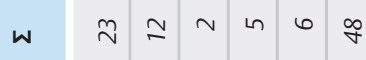
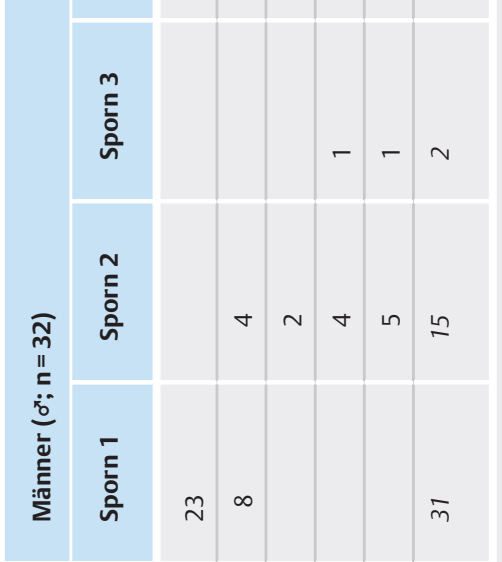

w

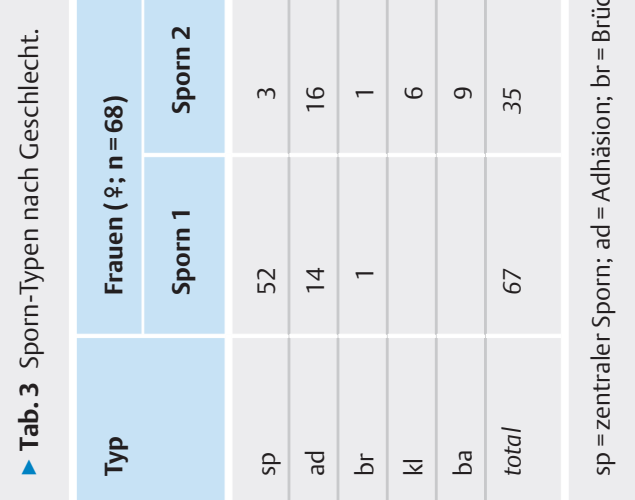

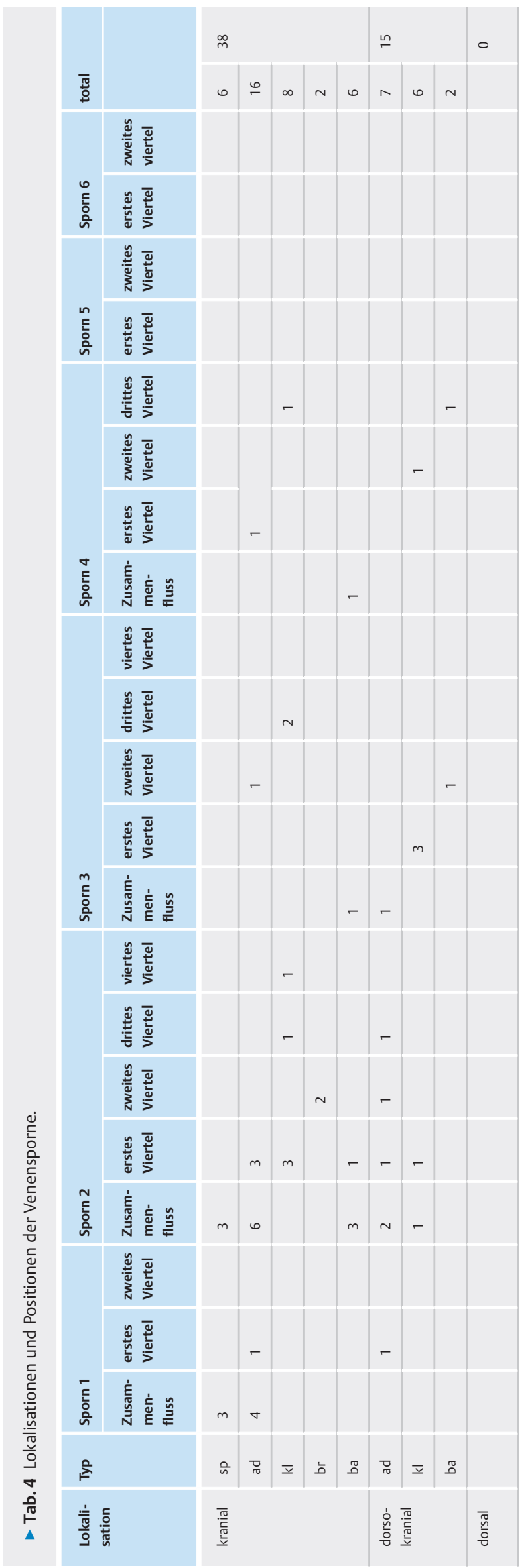




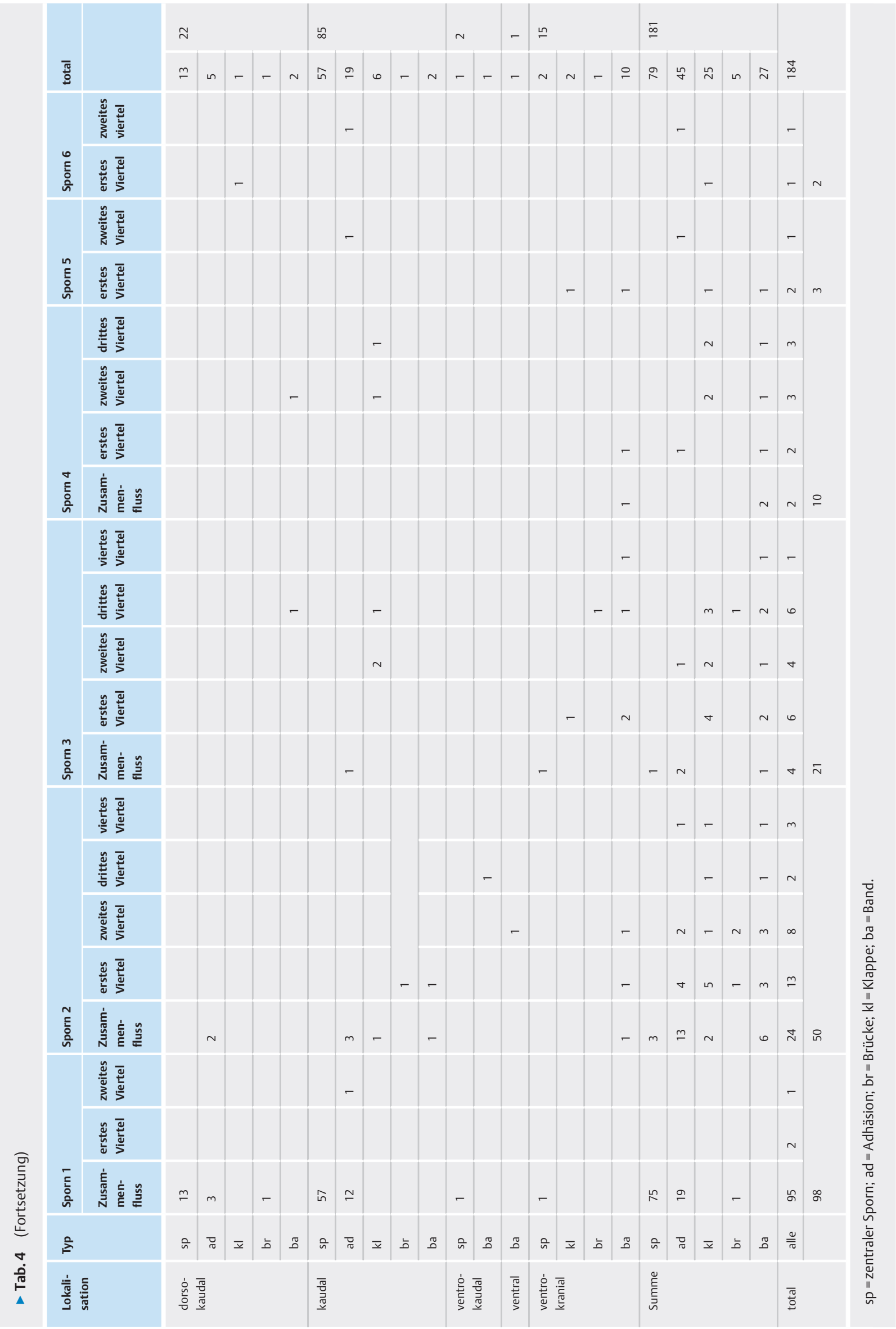




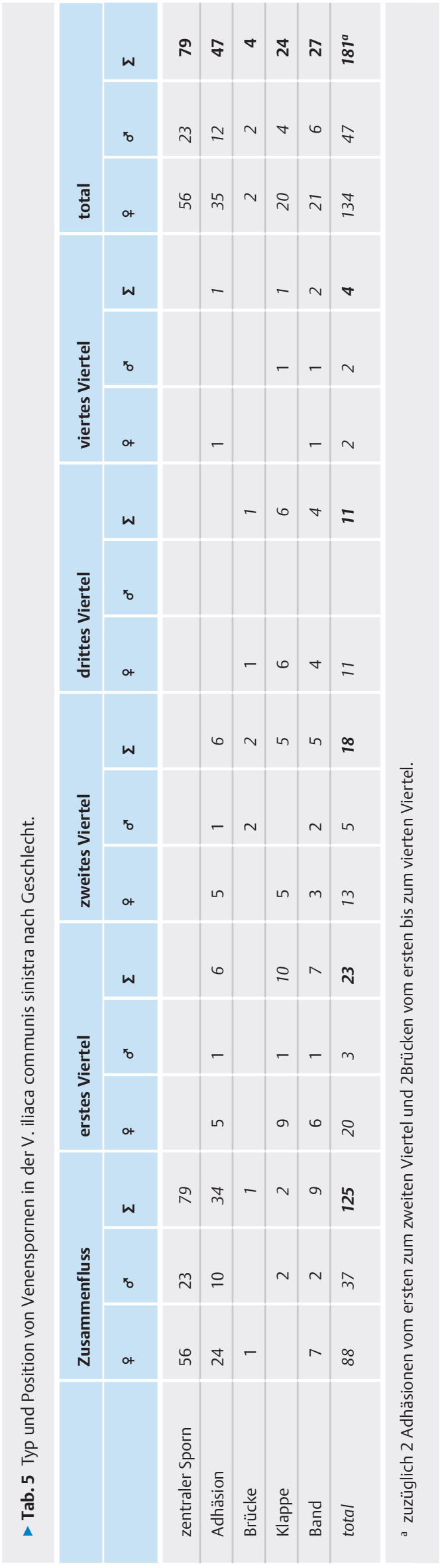

Bandes in den zentralen Vierteln nicht durch eine Kompression hervorgerufen wird, ist dies auch für das linke oder rechte Viertel unwahrscheinlich.

Ein kaudaler zentraler Sporn am Zusammenfluss der VICD und VICS, den wir in 57 Fällen gefunden haben, kann grundsätzlich auch einen strömungstechnischen Vorteil bieten, indem er eine Wirbelbildung des Blutes verhindert, ähnlich der Valva venae cavae inferioris im Atrium dextrum cordis. Er entspräche damit einer ostialen Klappe, die möglicherweise von beiden Iliakalvenen gemeinsam gebildet wird [20]. Zudem ist der Umfang - und damit die Querschnittsfläche - der VCIS am Zusammenfluss deutlich größer als in den distaleren Abschnitten.

May und Thurner schließen aus ihren histologischen Befunden, dass ein Venensporn scheinbar nur der Venenintima entstammt [7]. Sie gehen dabei davon aus, dass es durch die Pulsation der A. iliaca communis dextra zu einer sich immer wiederholenden Berührung der Venenwand kommt, die als chronischer Reiz zu einer Wucherung der Endothelien führt, die dann selbst wieder zu einer Verwachsung der beiden Wandungen und somit zur Spornbildung führen. Ihren eher einheitlichen Befunden stehen aber die stark variierenden Befunde von Mitsuoka und Mitarbeitern gegenüber [13]. Daraus muss geschlossen werden, dass es wahrscheinlich unterschiedliche Bildungsmechanismen geben könnte. Allerdings lassen unsere Ergebnisse mit zum Teil mehreren Venenspornen über die ganze VCIS hinweg verteilt darauf schließen, dass eine etwaige Kompression durch die A. iliaca communis dextra oder die A. iliaca communis sinistra [19] nicht für alle Venensporne verantwortlich sein kann.

In der weiteren Forschung ist mit der Zeit jedoch die Betonung auf das Vorhandensein von Venenspornen verloren gegangen, da sich die Untersucher*innen auf die Symptome der Patient*innen konzentrieren [21]. Demnach steht die Kompression der VICS durch die A. iliaca communis dextra im Vordergrund [e. g. ref. 22]. Diese - zumeist radiologischen - Studien unterliegen allerdings oftmals einem oder gar mehreren grundsätzlichen Irrtümern in der Bestimmung des Ausmaßes der Obstruktion. Es ist unbestritten, dass eine Kompression auch zu einer Obstruktion führt. Allerdings wird die Ausbildung von beeindruckenden Kollateralen außer Acht gelassen [11]. Zum anderen wird das Ausmaß der kompressionsbedingten Obstruktion oftmals überschätzt. So wurde etwa die „Stenose“ der VICS als Quotient der minimalen Durchmesser der VICS und der VICD dargestellt [23], außer Acht lassend, dass sich ein kreisförmiger Gefäßquerschnitt bei Kompression in einen annähernd elliptischen Querschnitt verwandelt. Legen wir die Daten der Studie von Carr und ihren Kolleg*innen [23] zugrunde, so besitzt eine unkomprimierte VCIS einen Durchmesser von 6,5 mm. Dies bedeutet einen Umfang von 20,4 mm und eine Querschnittsfläche von 33,2 mm². Auf Basis des Umfangs kann nun auch der Querschnitt einer komprimierten VICS näherungsweise errechnet werden. Nach Carr et al. hat eine komprimierte VICS einen minimalen Durchmesser von 4,0 mm. Damit beträgt der maximale Durchmesser einer Ellipse von 20,4 mm Umfang etwa 6,8 mm und die Querschnittsfläche beträgt dann etwa $27,0 \mathrm{~mm}^{2}$, also immerhin noch $81 \%$ der unkomprimierten VICS. Es liegt also keine wirkliche Stenose vor; die etwaige Flussbehinderung kann einerseits über die bereits beschriebene Kollateralenverstärkung, als auch ganz einfach durch eine lokale Fluss- 


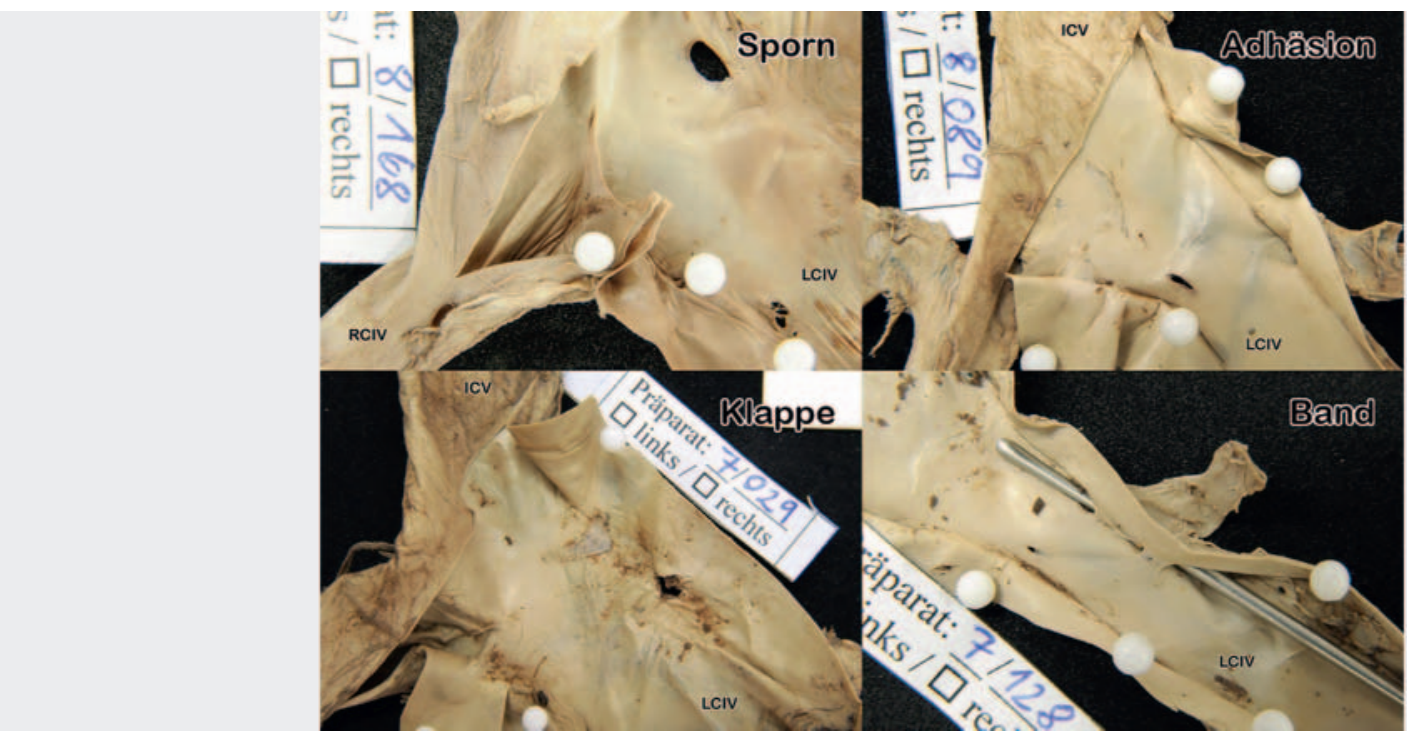

- Abb. 5 Beispiele der Spornbildungen. ICV=V. cava inferior; LCIV=V. iliaca communis sinistra; RCIV=V. iliaca communis dextra. 8/168: weiblich, 84 Jahre, zentraler Venensporn am Zusammenfluss; 8/089: weiblich, 83 Jahre, Adhäsionen am Zusammenfluss; 7/029: männlich, 80 Jahre, zentraler Venensporn und Klappe am Zusammenfluss; 7/128: weiblich 66 Jahre, zentraler Venensporn am Zusammenfluss und Band im ersten Viertel.

geschwindigkeitserhöhung ausgeglichen werden, womit das Volumen/Zeit konstant bleiben kann.

\section{Schlussfolgerungen}

Venensporne sind nach unseren Daten wesentlich häufiger als frühere Studien erwarten ließen. Neben den „klassischen“ zentralen Spornen finden sich Adhäsionen, brückenartige Strukturen, Klappen und bandartige Verbindungen der Venenwand. Mit Ausnahme der kaudalen zentralen Sporne am Zusammenfluss von VICD und VICS stellen sie grundsätzlich ein Strömungshindernis dar und können so einen prädisponierenden Faktor für eine tiefe Venenthrombembolie der VICS darstellen. Diese Strukturen finden sich zudem nicht nur am Zusammenfluss, sondern auch über die ganze Länge der aus der interkardinalen Anastomose entstandenen VICS. Demnach erscheint allerdings ein ursächlicher Zusammenhang mit einer Kompression durch die A. iliaca communis dextra unwahrscheinlich.

\section{Ethische Aspekte}

Die Körper wurden von Personen gespendet, die vor ihrem Tod ihre informierte Zustimmung zur Verwendung für wissenschaftliche und pädagogische Zwecke gegeben haben [16, 24].

Interessenkonflikt

Die Autorinnen/Autoren geben an, dass kein Interessenkonflikt besteht.

\section{Danksagung}

Wir danken Dr. ${ }^{\text {in }}$ Dr. in Romana Urbas, PhD, für ihre wertvollen Beiträge. Die Autoren möchten den Personen danken, die ihren Körper und ihr Gewebe für die Förderung von Bildung und Forschung gespendet haben.
Literatur

[1] Raju S, Darcey R, Neglén P. Unexpected major role for venous stenting in reflux disease: Symptom relief with partial correction of pathology. J Vasc Surg 2009; 49: S5 doi:10.1016/j.jvs.2009.02.143

[2] Virchow R. Thrombose und Embolie. Gefässentzündung und septische Infektion. In Gesammelte Abhandlungen zur wissenschaftlichen Medicin. Frankfurt am Main: Meidlinger Sohn \& Comp; 1862: 219-732

[3] Hach W, Hach-Wunderle V. Robert May (1912-1984) und die Phlebologie in seiner Zeit. Gefässchirurgie 2000; 5: 200-207. doi:10.1007/ PL00010595

[4] McMurrich JP. The occurrence of congenital adhesions in the common iliac veins, and their relation to thrombosis of the femoral and iliac veins. American Journal of the Medical Sciences 1908; 135: 342-346. doi:10.1097/00000441-190803000-00004

[5] Ehrich WE, Krumbhaar EB. A frequent obstructive anomaly of the mouth of the left common iliac vein. Am Heart J 1943; 26: 737-750. doi:10.1016/S0002-8703(43)90285-6

[6] Schilling W. Über Phlebosklerose, ihre Entstehungsweise und Ursachen. Im Anhang über Bildungsanomalien in der Vena iliaca communis und externa. Virchows Archiv für pathologische Anatomie und Physiologie und für klinische Medizin 1926; 262: 658-680. doi:10.1007| BF01996865

[7] May R, Thurner ]. Ein Gefäßsporn in der Vena iliaca communis sinistra als wahrscheinliche Ursache der überwiegend linksseitigen Beckenvenenthrombose. Z Kreislaufforsch 1956; 45: 912-917

[8] May R, Thurner ]. The cause of predominantly sinistral occurrence of thrombosis of the pelvic veins. Angiology 1957; 8: 419-427. doi: $10.1177 / 000331975700800505$

[9] Cockett FB, Thomas ML. The iliac compression syndrome. BJS (British Journal of Surgery) 1965; 52: 816-821. doi:10.1002/bjs.1800521028

[10] Negus D, Fletcher EW, Cockett FB et al. Compression and band formation at the mouth of the left common iliac vein. Br J Surg 1968; 55: 369374. doi:10.1002/bjs. 1800550510 
[11] Chermet J. Le syndrome de compression veineuse iliaque commune gauche. Anatomia Clinica 1979; 1: 347-355. doi:10.1007/BF01654711

[12] Pinsolle J, Drouillard J, Grenier F et al. Internal Arrangement of the Union between Iliac Vein and Inferior Vena-Cava. Anatomia Clinica 1982; 4 : 295-306. doi:10.1007/Bf01798559

[13] Mitsuoka H, Ohta T, Hayashi S et al. Histological study on the left common iliac vein spur. Ann Vasc Dis 2014; 7: 261-265. doi:10.3400/ avd.oa.14-00082

[14] Starck D. Embryologie - Ein Lehrbuch auf allgemein biologischer Grundlage. 3rd. Aufl. Stuttgart: Thieme; 1975

[15] Platzer W, Putz R, Poisel S. Ein neues Konservierungs- und Aufbewahrungssystem für anatomisches Material. Acta Anat (Basel) 1978; 102: 60-67

[16] McHanwell S, Brenner E, Chirculescu ARM et al. The legal and ethical framework governing Body Donation in Europe - A review of current practice and recommendations for good practice. Eur J Anat 2008; 12: $1-24$

[17] Konschake M, Brenner E. „Mors auxilium vitae“-Causes of death of body donors in an Austrian anatomical department. Ann Anat 2014; 196 : 387-393. doi:10.1016/j.aanat.2014.07.002
[18] Yagnik ]. PSPP a free and open source tool for data analysis. Voice Res 2014; $2: 73-76$

[19] Caggiati A. The left common iliac artery also compresses the left common iliac vein. J Vasc Surg 2011; 54: 56S-61S. doi:10.1016/j.jvs.2011.06.031

[20] Franklin KJ. Valves in Veins: An Historical Survey. Proc R Soc Med 1927; 21: 1-33. doi:10.1177/003591572702100101

[21] Toonder IM. The Myth of May Thurner. Phlebologie 2020; 49: 230-232. doi:10.1055/a-1213-0756

[22] Brinegar KN, Sheth RA, Khademhosseini A et al. Iliac vein compression syndrome: Clinical, imaging and pathologic findings. World J Radiol 2015; 7: 375-381. doi:10.4329/wjr.v7.i11.375

[23] Carr S, Chan K, Rosenberg J et al. Correlation of the diameter of the left common iliac vein with the risk of lower-extremity deep venous thrombosis. J Vasc Interv Radiol 2012; 23: 1467-1472. doi:10.1016/ j.jvir.2012.07.030

[24] Riederer BM, Bolt S, Brenner E et al. The legal and ethical framework governing Body Donation in Europe - 1st update on current practice. Eur J Anat 2012; 16: 1-21 